\title{
An Inclusive and Individualized Response for Victims of Crime with Disabilities: The Adult Advocacy Centers 'Model
}

\author{
Olivia Caldeira ${ }^{1}$, Leigha Shoup ${ }^{2}$ \\ Adult Advocacy Centers, United States
}

\begin{abstract}
This paper provides an overview to the Adult Advocacy Centers (AACs), the first centers in the world that will be equipped to provide holistic, accessible, and trauma-informed services to adult crime victims with disabilities in a universal and multi-sensory environment. The AACs will work in partnership with state, regional, and community agencies to coordinate a response that promotes the safety and well-being of all individuals. To provide these services, the AACs will facilitate multi-disciplinary teams (MDTs) within local communities, implementing a forensic protocol that optimizes the possibility that perpetrators will be prosecuted. The AACs plan to build 10 centers throughout the state of Ohio and create a mobile MDT that will travel to crime victims who are unable to visit a center. An exploration into this unique approach to victim services will assist participants in understanding best practices for survivors. This paper provides a greater understanding of the various needs of crime victims with disabilities and how the AACs' unique design and collaborative efforts will ensure equal accessibility to necessary investigative, prosecutorial, and treatment services when there are allegations of abuse and neglect. While it is absolutely important for everyone to have a voice, it is also important to teach others how to listen. People with disabilities who have been victims of abuse or neglect deserve to have their needs met in a holistic manner and remain at the center of the investigation and treatment plan.
\end{abstract}

Keywords: crime victims, disabilities, advocacy, accessibility

\section{Introduction}

People with disabilities are victimized at a higher rate than people without disabilities (Harrell, 2017). ${ }^{1}$ According to the Vera Institute of Justice, "the rate of violent victimization against people with disabilities, which includes simple assault in addition to serious violent crimes, has been at least twice that of people without disabilities, making people with disabilities one of

\footnotetext{
${ }^{1}$ Harrell, E. (2017). Crime Against Persons with Disabilities 2009-2015 - Statistical Tables. Bureau of Justice Statistics. Available: https://www.bjs.gov/content/pub/pdf/capd0914st.pdf
} 
the most harmed groups in the United States."2 Multiple factors can lead to people with disabilities being more vulnerable to victimization, including the following: isolation, reliance on perpetrator for assistance, fear of not being believed, fear of retribution, fear of loss of rights and independence, lack of awareness that what they are experiencing is abuse or a crime, culture of compliance, lack of communication options, lack of education regarding sexuality and healthy relationships, and limited knowledge or ability to contact help. The aforementioned factors, coupled with physical, procedural, and attitudinal barriers preventing crime victims with disabilities from accessing the necessary services and support to seek justice and heal, warrant critical attention and an effective solution. The Adult Advocacy Centers (AACs) seek to address the gaps in services and provide support throughout the criminal justice process and beyond for adult crime victims with disabilities.

\section{Supporting Research}

Statistics in the United States show that individuals with disabilities are more likely to experience abuse than people without disabilities (Harrell, 2017). ${ }^{3}$ A nationwide survey by the Disability Abuse Project (2013) reported that more than 70 percent of people with disabilities who were surveyed reported they had been victims of abuse. More than 63 percent of family members said their loved one with a disability had been a victim of abuse. Focusing exclusively on individuals with developmental disabilities, 62.5 percent reported that they had experienced abuse (Coleman, $2013)^{4}$ and are two and a half to 10 times more likely to experience abuse and neglect than their non-disabled peers (Kendall-Tackett, 2002). ${ }^{5}$ This data is also reflected in international studies. Victim Support, an organization in the UK, collected data in England and Wales and discovered that people with limiting disabilities or illnesses were three and a half times more likely to experience violence with injury than those without disabilities. ${ }^{6}$

Although the research indicates increased rates of abuse and violent crimes against adults with disabilities, there is still a scarcity of systemized data regarding abuse against those with disabilities due to multiple factors, like underreporting, different definitions of disability and abuse, and

\footnotetext{
${ }^{2}$ Smith, N., Harrell, S., and Judy, A. (2017). How Safe are Americans with Disabilities? The facts about violent crime and their implications. Vera Institute of Justice. Available:

https://www.vera.org/downloads/publications/How-safe-are-americans-with-disabilities-web.pdf

${ }^{3}$ Harrell, E. (2017). Crime Against Persons with Disabilities 2009-2015 - Statistical Tables. Bureau of Justice Statistics. Available: https://www.bjs.gov/content/pub/pdf/capd0914st.pdf

${ }^{4}$ Coleman, T. (2013). Statistics on Abuse Should Inform the Legislative Process. Available: https://disabilityandabuse.org/abuse-statistics.pdf

${ }^{5}$ Kendall-Tackett, K. (2002). The Health Effects of Childhood Abuse: Four Pathways by which Abuse can Influence Health. Child Abuse Neglect, 26(6-7), 715-729.

${ }^{6}$ Rossetti, P., Dinisman, T., and Moroz, A. (2016). Insight Report: An Easy Target? Risk factors affecting victimization rates for violent crime and theft. Available: https://www.victimsupport.org.uk/sites/default/files/VS\%20Insight\%20Report\%20-\%20An\%20easy\%20target.pdf ${ }^{7}$ Baladerian, N. and Coleman, T. (2013). Abuse of People with Disabilities: Victims and Their Families Speak Out. Available: https://disabilityandabuse.org/survey/survey-report.pdf
} 
methodological challenges, to name a few (Harrell, 2017; Baladerian and Coleman, 2013). ${ }^{7}$ Unfortunately, there are less services and support options for adult crime victims with disabilities than for those without disabilities. From interactions with first responders and law enforcement officers, prosecuting attorneys, judges and magistrates, to treatment and therapeutic opportunities for initial and ongoing support, crime victims with disabilities face significant challenges and barriers in order to receive the support they need and deserve. Analogous to the research trends cited earlier, national and international research reflects similar obstacles to receiving appropriate and accessible services. While people with disabilities in the United States are three times more likely than people without disabilities to experience violent crimes, only 13 percent received victim services. ${ }^{7}$ This disparity between increased need and less services is also evident in China, one of the first countries to ratify the UN Convention on the Rights of Persons with Disabilities (CRPD), which has created over 60 laws and regulations to protect the rights of people with disabilities. In spite of these proactive measures, people with disabilities are the least likely to receive and benefit from the legal aid services created to assist them. ${ }^{8}$ Correspondingly, in a study conducted in Ireland, multiple barriers were listed as factors preventing many crime victims with disabilities from participating and receiving services through the criminal justice system. ${ }^{910}$ Based on the findings gathered from national and international sources, the barriers and obstacles facing crime victims with disabilities are not unique to one particular region, but instead are cause for global concern.

In order to gain more perspective on the underlying issues that have created the conditions impeding people with disabilities from accessing the services and support they need after they have been a victim of a crime, it is necessary to draw attention to some of the key barriers they potentially face on a global level (but this is by no means an exhaustive list). Since abuse is about power and control, the same factors that are responsible for people with disabilities being at an increased risk of being abused and victimized are interlaced throughout attitudinal, physical/structural, and procedural obstacles and thus prevent them from receiving necessary support at multiple interaction points throughout the criminal justice process. ${ }^{11}$

\footnotetext{
${ }^{7}$ Harrell, E. (2017).

${ }^{8}$ United Nations Development Programme. (2016). Equal Access to Justice for Persons with Disabilities in China. Available: https://www.undp.org/content/dam/china/img/demgov/UNDP-CH-PublicationsDGEqual\%20Access\%20to\%20Justice\%20for\%20Persons\%20with\%20Disabilities\%20in\%20China(EN).pdf ${ }^{9}$ Edwards, C., Harold, G., and Kilcommins, S. (2012). Access to Justice for People with Disabilities as Victims of Crime in Ireland. Available: http://nda.ie/nda-files/Access-to-Justice-for-People-with-Disabilities-as-VictimsofCrime-in-Ireland1.pdf ${ }^{11}$ Ohio Victim Witness Association. (2018). Serving Survivors with Disabilities: An Advocate's Guide. Available: https://s3.amazonaws.com/ClubExpressClubFiles/732110/documents/OVWA_Serving_Survivors_w_Disabilities $\underline{2}$ 10 _web 395508998.pdf?AWSAccessKeyId=AKIA6MYUE6DNNNCCDT4J\&Expires=1581620770\&responsecontent disposition=inline \%3B\%20filename\%3DOVWA_Serving_Survivors_w_Disabilities_2018_web.pdf\&Signature=ST luIYw\%2BHd7sS\%2F3OZLyWBtt3yxo\%3D
} 
People with disabilities are often stigmatized and viewed from a perspective as being deficient in some form. This perspective results in attitudinal, physical/structural, and procedural barriers that deny many people with disabilities from being treated with the dignity and respect afforded those without disabilities. ${ }^{11}$ When it comes to allegations of abuse, crime victims with disabilities encounter attitudinal obstacles regarding knowledge and awareness, credibility, ability to respond to therapeutic measures, and a paternalistic sense of overprotection. Support persons, caregivers, guardians, and family members might carry some of these implicit biases and either fail to recognize signs and reports of abuse, not believe, or want to protect the person with disabilities from a potentially lengthy, retraumatizing, and unsuccessful criminal justice process. Law enforcement and investigative agents often lack specific forensic investigative training to effectively communicate and work with adult crime victims with disabilities. Like law enforcement officers, medical professionals might not have specific training and know best practices for conducting medical examinations for adult crime victims with disabilities.

Prosecutors, attorneys, magistrates, and judges lack disability-specific training concerning crime victims with disabilities, have limited knowledge of case law examples where people with disabilities were able to obtain justice, and might not know how to make the criminal justice process accessible. Finding counselors, therapists, and mental health professionals who are specifically trained to work with adult crime victims with disabilities can be a challenge, which then leads to a lack of effective therapeutic aftercare options for both individual and group therapies. Some communities offer support groups for crime victims, but accessibility and resources can be attitudinal and physical/structural obstacles for people with disabilities to be included and participate on an equal level.

Ohio is the first state in the United States to develop centers that will be equipped to address the needs of crime victims with disabilities by providing holistic, accessible, and trauma-informed services in a universally designed and multisensory environment. Ohio is also unique due to its robust system that was established over 60 years ago to provide services for individuals with developmental disabilities. Each of Ohio's 88 counties has its own county board of developmental disabilities and those 88 county boards are mandated through the state of Ohio's Department of Developmental Disabilities. County boards have an established infrastructure for investigating allegations of abuse, employ certified Investigative Agents (IAs) in every county, and utilize a state-of-the-art computerized database for reporting incidents of abuse. In order to gain a better understanding of the necessary services and supports for adult crime victims with disabilities, the Adult Advocacy Centers (AACs) conducted a needs assessment and received the most responses from county boards. This information was then compiled, summarized into a report, and published online through the AACs website. ${ }^{12}$ The needs assessment reflected national and international data regarding the lack of consistent and accessible services and indicated a critical need for a new approach. Additionally, the assessment signaled the necessity for further research and data collection to better identify and understand the needs of adult crime victims with disabilities.

\footnotetext{
${ }^{11}$ Edwards et. al. (2012).

12 www.adultadvocacycenters.org
} 
For the sake of space and time, this section will highlight some of the key points gathered from the county boards of developmental disabilities. Regarding the initial stages of interaction between crime victims with disabilities and first responders, the needs assessment suggested there were some areas where the processes are working, but more areas where additional improvements are necessary. Although there is a 24-hour crisis line available to report abuse, many counties reported an absence of a specified team designated to respond collaboratively to allegations of abuse. Additionally, many responses pointed to a deficit in effective multidisciplinary teams (MDTs) and inter-agency collaboration. The county board responses indicated that law enforcement and hospitals are promptly notified and routinely involved when there has been an allegation of abuse. However, many respondents reported a lack of forensic interviewing protocol geared towards adults or individuals with developmental disabilities, requiring crime victims to be interviewed multiple times by several different agencies, potentially resulting in repeat trauma. Responses revealed poor linkage to the county prosecutor, resulting in fewer cases being taken to court or to a grand jury due to a perceived lack of witness credibility or lack of comprehension and an unwillingness to participate in the process. These barriers prevent more crime victims with disabilities going to court and accessing the services of the criminal justice system, thereby enabling more perpetrators to evade prosecution and possible sentencing. ${ }^{13}$

In addition to significant needs for more specially-trained forensic interviewers and protocols geared towards crime victims with disabilities, inter-agency collaborative efforts, and stronger support and increased accessibility for victims as they try to navigate the criminal justice system, the needs assessment highlighted a lack of support and services for treatment and aftercare for adult crime victims with disabilities. Many of the counties responded that they referred crime victims with disabilities to trauma-informed mental health professionals, but these activities were completed on a case-by-case basis without the benefit of a guiding protocol. Counties overwhelmingly responded that there were no specific support groups for crime victims with disabilities. In counties where support groups existed that could potentially be appropriate and helpful, accessibility remained a challenge.

When asked what they thought was the biggest barrier/obstacle to providing crime victim services to people with disabilities in their county, over half the responses pointed to lack of resources. Although the state does provide some oversight and funding opportunities [check this for accuracy], counties have vastly different resources and budgets, which greatly impacts the quality of support and services crime victims with disabilities are able to access. A quarter of the responses identified lack of collaboration as the biggest barrier. Others mentioned attitudinal barriers of disregarding people with disabilities - namely, when allegations of abuse made by people with disabilities were not taken seriously or individuals with disabilities were not considered capable of testifying or being a witness.

At the end of the needs assessment, respondents were asked what they believed would be the best way to support their county in creating new programs and services focused on crime victims with

\footnotetext{
${ }^{13}$ https://www.adultadvocacycenters.org/wp-content/uploads/2019/11/Needs_Assessment 2019.pdf
} 
disabilities. Almost half answered more collaboration and support, and others recommended more programs, resources, training, and education. There was also a clear and consistent incentive crossing all counties to initiate an investigative protocol that would enhance the opportunity for prosecution. Overall, the assessment results validated what had long been suspected-Ohio currently lacks adequate support services, prosecution, and appropriately-trained forensic interviewers for adult victims of crime with disabilities. With local and statewide support, Ohio is committed to working toward equality in the field of victim services. The AACs will be the embodiment of this commitment.

\section{The Adult Advocacy Centers (AACs) Model}

The Adult Advocacy Centers' vision is to serve adults with disabilities who are alleged victims or witnesses of abuse, maltreatment or neglect. The AACs' staff and partner agencies are expertly trained to meet the needs of every individual in a holistic manner, ensuring they are the center of the investigation and treatment plan. The AACs' model consists of four key components: the centers, partnerships, trainings, and ongoing supp. Through collaborative efforts and a clear commitment to incorporating best practices, the Adult Advocacy Centers will be effecting positive systemic changes at multiple levels to create lasting benefits for adult crime victims with disabilities. As AACs' Executive Director, Katherine Yoder, has succinctly stated, "Adult Advocacy Centers were not created to help crime victims with disabilities find their voice, but to teach everyone else how to listen."14

\subsection{Centers}

The AACs are designing and building 10 centers across the state of Ohio with disabilityspecific accommodations that exceed ADA requirements by integrating a universal, multi-sensory, and trauma-informed design. These centers will allow all adults with disabilities to access victim services in an equal, inclusive, and accessible manner, regardless of where they live in the state. Each center will be identical on the inside but unique on the outside to ensure quality and standardization of services. The future sites and locations were selected considering the following factors: population of people with disabilities; proximity to certain state-run developmental centers, psychiatric hospitals, and existing Child Advocacy Centers; notoriously underserved rural areas; and regional sites in counties where those in neighboring counties would be able to travel to the centers relatively easily (see Figure 1). In addition to the brick and mortar centers, there will be a mobile RV (Recreational Vehicle) unit equipped with state-of-the-art equipment to bring services to areas for victims of crime who are unable to come to one of the centers. A traveling Multi-Disciplinary Team (MDT) will also be available for specific cases where the victim of crime is unable to travel (i.e., facility-based, high medical needs, etc.). This team would be designed to respond to the specific needs of the individual and bring together people who would be able to provide as many services as possible. The mobile MDT would likely include a specially-trained

\footnotetext{
${ }^{14}$ Yoder, K. (10/24/2019). Personal communication with Adult Advocacy Centers' Executive Director.
} 
forensic interviewer, a specially-trained forensic medical examiner (if needed), a victim advocate, mental health support professional, prosecuting attorney, and law enforcement officer.

Figure 1: Map of AACs with other resources

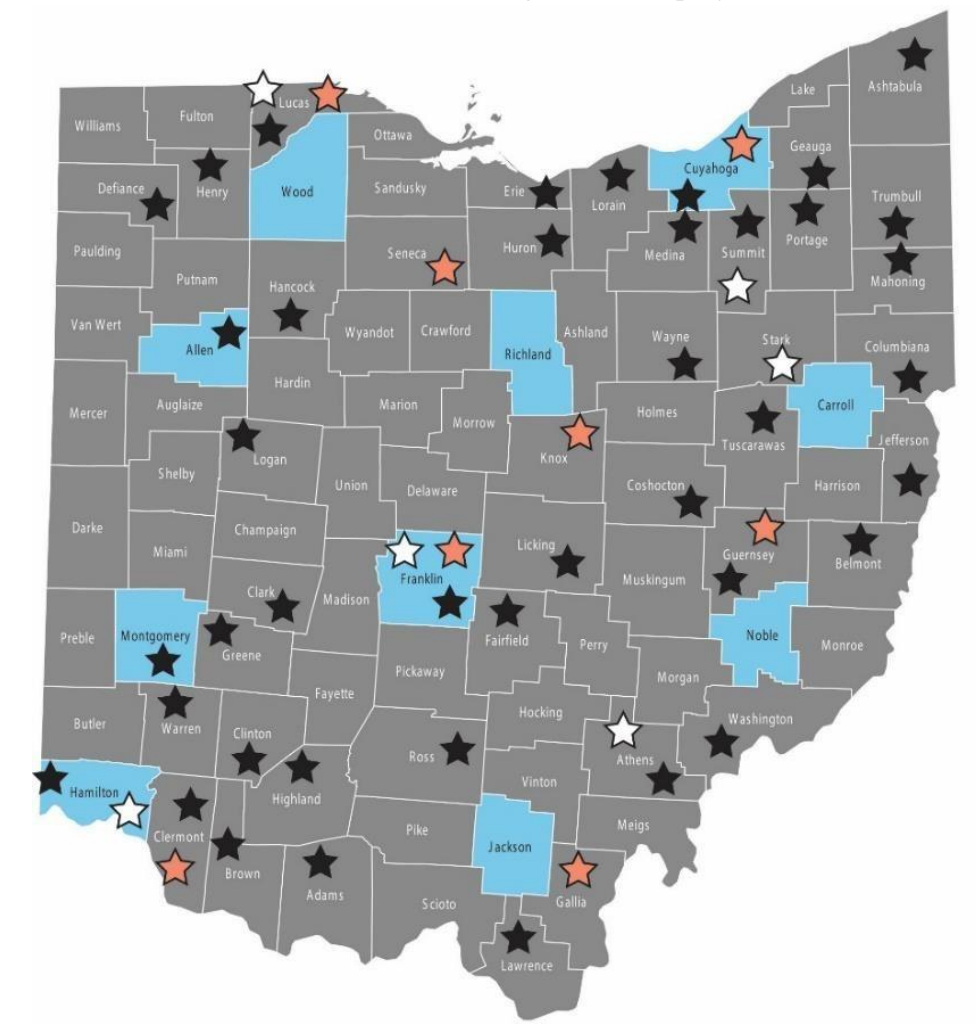

Counties in blue indicate planned sites for Adult Advocacy Centers

Counties with Child Advocacy

Centers, including satellite offices and planned sites

$\approx$ Counties with State

Developmental Centers

$\hat{\omega}$ Counties with State

Psychiatric Hospitals

Source: https://www.adultadvocacycenters.org/wp-

content/uploads/2019/11/AACs_Map_and_List_with_Related_Services.pdf

\subsection{Partnerships}

The second component of the AACs model is the commitment to foster collaboration among community, regional, and state agencies to coordinate a response that promotes the safety and wellbeing of all individuals. To provide these services, the AACs will facilitate Multidisciplinary Teams (MDTs) within local communities. The AACs' MDTs include the following: law enforcement agencies, investigative agents, medical staff, local county boards of developmental disabilities, mental health agencies, AACs' staff counselors, victim service agencies/advocates, prosecutors, victim's support person/guardian (as needed), adult protective agencies, and other agencies, as needed. By coordinating the MDTs and promoting inter-agency collaboration, the AACs will provide the framework, training, and facilitation necessary to ensure that crime victims with disabilities are supported in a comprehensive and trauma-informed manner. Some of these services include the following: 
- Forensic interviews by highly-trained and skilled investigators

- Forensic medical exams conducted by forensic nurses who specialize in working with crime victims with disabilities

- Connection to law enforcement agencies

- Early connection to prosecutors

- Mental health screenings, consultations, and services

- Connection to disability-specific agencies and organizations

- Victim services, advocacy, court accompaniment, case updates, and notifications

- Information Technology (IT) case management system specifically for the AACs

- 24/7 emergency medical responses (at local emergency departments)

- Mobile forensic interview teams for individuals in locations unable to reach the AACs

- Monthly case review meetings for MDTs

- Ongoing training to better serve crime victims with disabilities

- Peer review to help forensic interview team members stay up-to-date on current best practices

\subsection{Training}

The third component of the AACs' model is training. Training centers will be included in each center for people who are employed to provide services for crime victims with disabilities and people with disabilities who have been victimized. National experts in the fields of abuse, forensic services, specific disabilities, support services, medical, criminal justice, clinical services, and advocacy will be partnered with as consultants. The AACs are currently providing unique forensic interview training, entitled Project FIND, for various MDT members across the state at no cost to the participants. The Advanced Forensic Interviewing for Individuals with Disabilities certificate training teaches forensic interviewing best practices and makes considerations and adaptations to account for each individual's disability, mental health disability, assistive technology, language capacity, and cultural background. This training establishes and implements a protocol that is legally defensible to assist in the prosecution of crimes. Ohio is setting the standard for victims of crime with disabilities to accurately tell their stories in whatever way they communicate and take that evidence through the criminal justice system. Committed to the inclusion and perspectives of those the AACs will be serving, people with disabilities, including survivors, will be consulted to develop accessible resources, trainings, and educational programs and materials specifically for victims of crime.

\subsection{Supports}

The fourth element of the AACs' model is the commitment to providing ongoing supports. Ensuring that crime victims with disabilities receive continuous support, whether through individual, group, or other counseling and therapeutic methods, is critical in victim advocacy 
services. In response to the needs assessment, the AACs will address systemic gaps by working with self advocates and local agencies and organizations to create support groups that are accessible, holistic, and trauma-informed, in order to meet the individualized needs of those seeking additional support. The AACs are currently compiling information about local and statewide self-advocacy groups in Ohio to create the most up-to-date list of self-advocacy resources for people with disabilities. In collaboration with the Ohio Self Determination Association (OSDA), the Ohio Developmental Disabilities Council, and People First of Ohio, the AACs are creating an online resource for self-advocates. The AACs are also collaborating with self-advocates to develop an accessible guidebook on how to be a self-advocate. This guidebook, based on feedback from self-advocates, will offer different definitions of self-advocacy and how to connect with the self-advocacy community, for those who are interested.

\section{Conclusion}

National and international research demonstrates the alarming rate at which people with disabilities are abused and the lack of services and trauma-informed protocols available for such victims. In 2019, the AACs conducted their own needs assessment to learn more about services and supports for adult crime victims with disabilities in Ohio. The AACs received a comprehensive response from county boards, which was extremely informative and appreciated. These responses verified the need for the AACs' model and clearly showed how dedicated county boards are to providing supports for individuals who have been abused. It is evident that county boards take allegations of abuse seriously and take actions to protect individuals with disabilities from future abuse.

Currently, when there is an allegation of abuse, law enforcement is consistently notified by county boards, and what follows this notification varies by county board, law enforcement jurisdiction, and prosecutor. At times, the county board is not involved with the investigation until law enforcement notifies the county board that they will not take the case. In a minority of counties, joint investigations between law enforcement and county boards occur. Training for law enforcement officers does not often go into great detail on how to investigate when the victim has a disability. This results in unintended consequences and discrimination as allegations of abuse are not always investigated properly or in a timely manner. This practice would cease if the AACs' model was utilized. The AACs' model would ensure that an inclusive and holistic approach was utilized to conduct timely forensic interviews and medical exams following an allegation of abuse.

Lastly, the absence of a forensic interview hinders the potential prosecution of abuse cases. Not only would the consistent implementation of a forensic protocol result in a greater number of prosecutable cases, investigators would receive proper and consistent training. To address these concerns, the AACs are in the process of providing additional forensic interview training and will be creating a prosecutor's guidebook.

Based on the needs assessment responses from county boards, which reflect national and international trends pointing to a lack of comprehensive and inclusive services for people with disabilities from the time of reporting through the criminal justice system process, it is evident that 
something must be done to address the needs of people with disabilities who have been victimized. By providing inclusive, accessible, and trauma-informed services, the Adult Advocacy Centers will ensure that adult crime victims with disabilities receive the support they need. It is the hope of the AACs that through incorporating collaborative partnerships, focusing highly-specialized personnel in each center who are able to provide individualized care based on each person's needs, and working with communities to foster supportive relationships, adult crime victims with disabilities will have greater chances at getting the justice they seek and the paths to recovery and resiliency they deserve.

\section{References}

Adult Advocacy Centers. (2019). Needs Assessment: Fall 2019. Available: https://www.adultadvocacycenters.org/current-work/\#needs

Baladerian, N. and Coleman, T. (2013). Abuse of People with Disabilities: Victims and Their Families Speak Out. Available: https://disabilityandabuse.org/survey/survey-report.pdf

Coleman, T. (2013). Statistics on Abuse Should Inform the Legislative Process. Available: https://disabilityandabuse.org/abuse-statistics.pdf

Edwards, C., Harold, G., and Kilcommins, S. (2012). Access to Justice for People with Disabilities as Victims of Crime in Ireland. Available: http://nda.ie/nda-files/Access-toJustice-for-People-with-Disabilities-as-Victims-of-Crime-in-Ireland1.pdf

Harrell, E. (2017). Crime Against Persons with Disabilities 2009-2015 - Statistical Tables.

Bureau of Justice Statistics. Available: https://www.bjs.gov/content/pub/pdf/capd0914st.pdf

Kendall-Tackett, K. (2002). The Health Effects of Childhood Abuse: Four Pathways by which Abuse can Influence Health. Child Abuse Neglect, 26(6-7), 715-729.

Ohio Victim Witness Association. (2018). Serving Survivors with Disabilities: An Advocate's Guide. Available:

https://s3.amazonaws.com/ClubExpressClubFiles/732110/documents/OVWA_Serving_S urvivors_w_Disabilities_2018_web_395508998.pdf?AWSAccessKeyId=AKIA6MYUE6 DNNNCCDT4J\&Expires $=1581620770 \&$ response-contentdisposition=inline \%3B\%20filename\%3DOVWA_Serving_Survivors_w_Disabilities_20 18 web.pdf\&Signature $=$ STluIYw\%2BHd7sS\%2F3OZLyWBtt3yxo\%3D

Rossetti, P., Dinisman, T., and Moroz, A. (2016). Insight Report: An Easy Target? Risk factors affecting victimization rates for violent crime and theft. Available:

https://www.victimsupport.org.uk/sites/default/files/VS\%20Insight\%20Report\%20\%20A n\%20easy\%20target.pdf

Smith, N., Harrell, S., and Judy, A. (2017). How Safe are Americans with Disabilities? The facts about violent crime and their implications. Vera Institute of Justice. Available: https://www.vera.org/downloads/publications/How-safe-are-americans-withdisabilitiesweb.pdf 
$2^{\text {nd }}$ International Conference on

SOCIAL SCIENCES IN THE 21ST CENTURY

27 - 29 MARCH , 2020

OXFORD, UNITED KINGDOM

United Nations Development Programme. (2016). Equal Access to Justice for Persons with Disabilities in China. Available:

https://www.undp.org/content/dam/china/img/demgov/UNDP-CH-Publications-

DGEqual\%20Access\%20to\%20Justice\%20for\%20Persons\%20with\%20Disabilities\%20i n\% 20China(EN).pdf

Yoder, K. (2019). Personal communication (October 24, 2019). 